\title{
A roadmap for planetary caves science and exploration
}

\begin{abstract}
To the Editor - 2021 is the International Year of Caves and Karst. To honour this occasion, we wish to emphasize the vast potential embodied in planetary subsurfaces. While researchers have pondered the possibility of extraterrestrial caves for more than 50 years, we have now entered the incipient phase of planetary caves exploration. Caves are important because they provide records of a planetary body's geological, meteorological and environmental history. On Mars, this may include evidence of past or even present microbial life. For the Moon and Mars, caves could protect human explorers from the harmful and inhospitable surface environment.
\end{abstract}

Our knowledge of planetary caves varies from body to body. Earth represents the most advanced level of exploration, but many unanswered questions remain (for example, a complete inventory of terrestrial caves is lacking). Beyond Earth, identification of possible caves is most advanced for the Moon and Mars ${ }^{1}$, with hundreds of documented candidate cave entrances and several proposed cave mission concepts. For other planetary bodies, potential subsurface access points (SAPs) have been identified, although confirmation of cave entrances has been hampered by our inability to sufficiently resolve SAP interiors (that is, the lack of off-nadir viewing platforms). To date, the community has catalogued 2,660 SAPs on eight planetary bodies (excluding Earth) across our Solar System (Fig. 1). Additionally, numerous satellites containing features associated with tectonism and cryovolcanism will require further scrutiny; thus, the number of SAPs and planetary bodies supporting these features will increase over time.

To systematically advance planetary caves exploration, we propose this roadmap composed of three conceptual phases:

(1) identification (orbital assets), (2)

characterization (surface operations) and (3) exploration (subsurface operations).

1. Identification. Thus far, most planetary cave entrances, skylights and collapse pits have been found by using standard remote context imaging ${ }^{2}$. On Earth, cave entrances can be identified via a combined thermal, visible and lidar approach. Such strategies should be further refined and expanded to detect caves on other planetary bodies. A

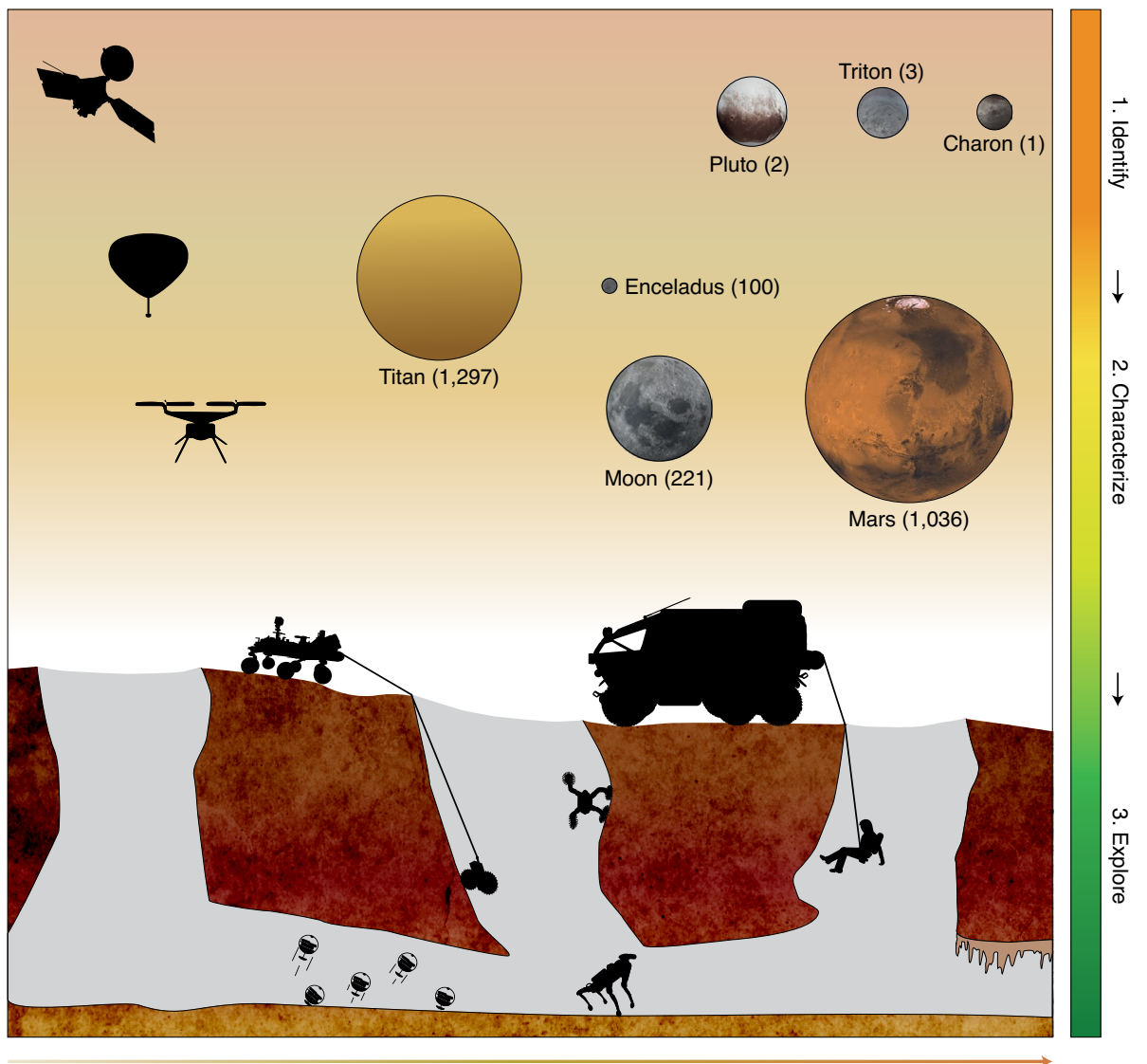

Progression of exploration

Fig. 1 | Planetary bodies are positioned within their respective research stage. Numbers in parentheses indicate total number of potential SAPs. Barring the Moon and Mars, most planetary bodies remain within the identification stage. Given the number of potential SAPs, Titan and Enceladus fall between investigation and characterization. Orbiter and suborbital balloon (for bodies with atmospheres) identification and aerial drone characterization (and possibly entrance examination) advances from top to bottom. Robotic and ultimately in situ human exploration of planetary caves occurs principally along a continuum from left to right (but is presently applicable only to the Moon and Mars). Hopping microbots and single-axle-tethered and limbed rovers are clustered together. While these robotic platforms are expected to perform similarly, robotics will be driven by mission requirements and science objectives $^{14-16}$. Credit: NASA / JPL-Caltech (images).

combination of these techniques with orbital subsurface geophysical methods including radar and gravimetrics ${ }^{3}$ could provide optimal advances in cave identification. However, to conduct a broader, Solar System-wide inventory of cave candidate entrances, additional orbiting spacecraft with sensors capable of accurately resolving these features is needed.

2. Characterization. Prior to the selection of an exploration target, candidate cave entrances must be thoroughly evaluated. High-resolution and oblique-angle imagery should be acquired for promising lunar and Martian cave candidates, that imagery systematically examined, and the features rank ordered by scientific importance. Additionally, current and future assets such as the Mars Ingenuity-like helicopters and Titan's Dragonfly (planned for 2034) and other proposed missions (for example, NASA's Moon Diver and the European Space 
Agency's lunar caves mission) could be used to confirm and/or examine scientifically interesting SAPs in situ. Surface missions can map cave geometries around the entrance and potentially define cave extent and volume if equipped with ground-penetrating sensors ${ }^{4}$. Resulting mapped cave architectures and hazards will inform mission planning and help reduce mission risk.

3. Exploration. Investment in the long lead-time robotic technologies is required to ultimately explore planetary caves. Various mission concepts have been proposed including limbed robots ${ }^{5}$, flying robot swarms $^{6}$, tethered rovers ${ }^{7}$, microbot swarms ${ }^{8}$, and deployable stationary payloads 9 . Each platform has unique capabilities and limitations, and selection will depend on cave structure and scientific objectives.

Robotic and artificial intelligence (AI) technologies for cave exploration have matured significantly over the last decade ${ }^{10}$. These include mobility in cave terrains, autonomous navigation, node-to-node communication, and sample site selection (for life detection and habitability assessments) in aphotic conditions. While significant technological advancements have been made, several additional engineering challenges remain - especially power, access and high-altitude entry descent and landing (EDL). Power will entail bundling within a tether, alternative internal power sources (for example, fuel cells), or recharging by returning to the surface. Navigating complex cave architectures will involve further AI development. On Mars, the most compelling SAPs examined thus far occur at high altitudes ${ }^{11,12}$. Landing at altitude will require either new pinpoint EDL methods or the capability to conduct a long-distance traverse from low- to high-altitude regions.

Planetary caves science has the potential to significantly expand over the next decade $^{13}$. On Earth, analogue studies and technological research and development will be imperative. The advent of aerial drones for bodies with atmospheres is a potential game changer; these systems could be used for both detection and entrance characterization. For rovers, spaceflight-qualified instruments capable of resolving (and characterizing) cave entrances and internal structure will be indispensable.

For the Moon and Mars, a mission in the near term (years to decades) is achievable, given appropriate investment in robotic development. Specifically, to reach the technological maturity required, the platforms discussed here should be developed to flight-qualified status. By applying this roadmap and advancing these key technologies (as well as site characterizations), we will be able to investigate the planetary subsurface - one of the most promising potentially habitable environments to search for evidence of life. This, in turn, will help foster the technological developments required for human exploration and habitation of caves on the Moon and Mars.

Timothy N. Titus (D) $1{ }^{凶}$, J. Judson Wynne (D) 2 , Michael J. Malaska (D) ${ }^{3 凶}$,

Ali-akbar Agha-Mohammadi ${ }^{3}$,

Peter B. Buhler ${ }^{4}$, E. Calvin Alexander ${ }^{5}$,

James W. Ashley ${ }^{3}$, Armando Azua-Bustos ${ }^{6,7}$ Penelope J. Boston ${ }^{8}$, Debra L. Buczkowski(D), Leroy Chiao ${ }^{10}$, Glen E. Cushing ${ }^{1}$, John DeDecker ${ }^{11}$, Pablo de León ${ }^{12}$, Cansu Demirel-Floyd ${ }^{13}$, Jo De Waele ${ }^{14}$, Alberto G. Fairén (D) 6,15, Amos Frumkin ${ }^{16}$, Gary L. Harris ${ }^{12}$, Heather Jones ${ }^{17}$,

Laura H. Kerber ${ }^{3}$, Erin J. Leonard ${ }^{3}$, Richard J. Léveillé18,19, Kavya Manyapu12, Matteo Massironi ${ }^{20}$, Ana Z. Miller ${ }^{21,22}$, John E. Mylroie ${ }^{23}$, Bogdan P. Onac (D)24,25, Scott Parazynski ${ }^{26}$, Cynthia B. Phillips (D) ${ }^{3}$ Charity M. Phillips-Lander (D) 27,

Thomas H. Prettyman (D) ${ }^{4}$, Haley M. Sapers ${ }^{28}$, Francesco Sauro ${ }^{14}$, Norbert Schorghofer (D)4, Dirk Schulze-Makuch 29,30,31,

Jennifer E. Scully (D) ${ }^{3}$, Kyle Uckert ${ }^{3}$, Robert V. Wagner ${ }^{32}$, William L. Whittaker ${ }^{17}$, Kaj E. Williams ${ }^{1}$ and Uland Y. Wong ${ }^{8}$ ${ }^{1}$ US Geological Survey, Astrogeology Science Center, Flagstaff, AZ, USA. ${ }^{2}$ Department of Biological Sciences, Northern Arizona University, Flagstaff, AZ, USA. ${ }^{3}$ NASA Jet Propulsion Laboratory, California Institute of Technology, Pasadena, CA, USA. ${ }^{4}$ Planetary Science Institute, Tucson, AZ, USA. ${ }^{5}$ Earth \& Environmental Sciences Department University of Minnesota, Minneapolis, MN, USA. ${ }^{6}$ Centro de Astrobiología, CSIC-INTA, Madrid, Spain. ${ }^{7}$ Instituto de Ciencias Biomédicas, Facultad de Ciencias de la Salud, Universidad Autónoma de Chile, Santiago, Chile. ${ }^{8}$ NASA Ames Research Center, Moffett Field, CA, USA. ${ }^{9}$ Planetary Exploration Group, Johns Hopkins University Applied Physics Laboratory, Laurel, MD, USA. ${ }^{10}$ Department of Mechanical Engineering, Rice University, Houston, TX, USA. ${ }^{11}$ Center for Mineral Resources Science, Colorado School of Mines, Golden, CO, USA.

${ }^{12}$ Human Spaceflight Laboratory, Department of Space Studies, University of North Dakota, Grand Forks, ND, USA. ${ }^{13}$ School of Geosciences, University of Oklahoma, Norman, OK, USA. ${ }^{14}$ Dipartimento di Scienze Biologiche, Geologiche e Ambientali, Università di Bologna, Bologna, Italy. ${ }^{15}$ Department of Astronomy, Cornell University, Ithaca, NY, USA. ${ }^{16}$ Institute of Earth Sciences, The Hebrew University of Jerusalem, Jerusalem, Israel. ${ }^{17}$ Robotics Institute, Carnegie Mellon University, Pittsburgh, PA, USA. ${ }^{18}$ Department of Earth \& Planetary Sciences, McGill University, Montreal, Quebec, Canada. ${ }^{19}$ Geosciences Department, John Abbott College, Ste-Anne-de-Bellevue, Quebec, Canada. ${ }^{20}$ Dipartimento di Geoscienze, Università degli Studi di Padova, Padova, Italy. ${ }^{21}$ Laboratório HERCULES, University of Évora, Évora, Portugal. ${ }^{22} I n s t i t u t o ~ d e$ Recursos Naturales y Agrobiología, Consejo Superior de Investigaciones Cientificas, Seville, Spain. ${ }^{23}$ Department of Geosciences, Mississippi State University, Starkville, MS, USA. ${ }^{24}$ School of Geosciences, University of South Florida, Tampa, FL, USA. ${ }^{25}$ Emil G. Racoviță Institute, Babes-Bolyai University, Cluj-Napoca, Romania. ${ }^{26}$ Fluidity Technologies, Inc., Houston, TX, USA. ${ }^{27}$ Southwest Research Institute, San Antonio, TX, USA. ${ }^{28}$ Department of Earth and Space Science and Engineering, York University, Toronto, Ontario, Canada. ${ }^{29}$ Astrobiology Research Group, Center for Astronomy and Astrophysics, Technische Universität Berlin, Berlin, Germany. ${ }^{30}$ German Research Centre for Geosciences, Section Geomicrobiology, Potsdam, Germany. ${ }^{31}$ Department of Experimental Limnology, Leibniz-Institute of Freshwater Ecology and Inland Fisheries, Stechlin, Germany. ${ }^{32}$ School of Earth and Space Exploration, Arizona State University, Tempe, AZ, USA.

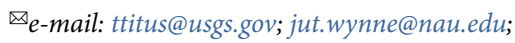
michael.j.malaska@jpl.nasa.gov

Published online: 15 June 2021

https://doi.org/10.1038/s41550-021-01385-1

References

1. Sauro, F. et al. Earth-Sci. Rev. 103288 (2020)

2. Malaska, M. J. Icarus 344, 113764 (2020).

3. Chappaz, L. et al. Geophys. Res. Lett. 44, 105-112 (2017).

4. Torrese, P. et al. Icarus 357, 114244 (2021).

5. Parness, A. et al. In Proc. 2017 IEEE International Conference on Robotics and Automation (ICRA) 5467-5473 (IEEE, 2017).

6. Dubowsky, S. et al. AIP Conf. Proc. 746, 1449 (2005).

7. Nesnas, I. A. D. et al. J. Field Robot. 29, 663-685 (2012).

8. Kesner, S. B., Plante, J.-S., Boston, P. J., Fabian, T. \& Dubowsky, S. In Proc. 2007 IEEE International Conference on Robotics and Automation 4893-4898 (IEEE, 2007).

9. Dille, M. et al. In Proc. 2020 IEEE Aerospace Conference https://doi.org/10.1109/AERO47225.2020.9172595 (IEEE, 2020).

10. Agha-Mohammadi, A. et al. J. Field Robot. (in the press).

11. Cushing, G. E. J. Cave Karst Stud. 74, 33-47 (2012).

12. Kearney, M. L., Wynne, J. J., Cushing, G. E., Bardabelias, N. M. \& Barlow, N. G. In Proc. 52nd Lunar and Planetary Science Conference LPI Contribution No. 2548 (LPI, 2021).

13. Titus, T. N., Phillips-Lander, C. M., Boston, P. J., Wynne, J. J. \& Kerber, L. Planetary cave exploration progresses. Eos https://doi. org/10.1029/2020EO152045 (2020).

14. Porco, C., DiNino, D. \& Nimmo, F. Astron. J. 148, 45 (2014).

15. Wagner, R. V. \& Robinson, M. S. In Proc. 52nd Lunar and Planetary Conference abstr. 2530 (LPI, 2021).

16. Cushing, G. E. Mars Global Cave Candidate Catalog (NASA, PDS, 2016); https://go.nature.com/2SwrqT0

\section{Acknowledgements}

Part of this research was conducted at the Jet Propulsion Laboratory, California Institute of Technology, under a contract with the National Aeronautics and Space Administration (80NM0018D0004). Images of planetary bodies were adapted from three-dimensional models available on NASA's Solar System Exploration website. Satellite and rover silhouettes were rendered and modified from images of NASA's Cassini and Perseverance spacecraft, respectively.

Author contributions

T.N.T., J.J.W. and M.J.M. share senior authorship. A.A. and P.J.B. developed the robotics section, while P.B.B. assisted with figure development. All other authors contributed equally. 